Fedoseeva O. V. Morphological changes in lung tissue in case of blunt abdominal trauma, massive blood loss, and ischemiareperfusion of the limb. Journal of Education, Health and Sport. 2020;10(8):589-597. eISSN 2391-8306. DOI http://dx.doi.org/10.12775/JEHS.2020.10.08.070

https://apcz.umk.pl/czasopisma/index.php/JEHS/article/view/JEHS.2020.10.08.070

https://zenodo.org/record/4437323

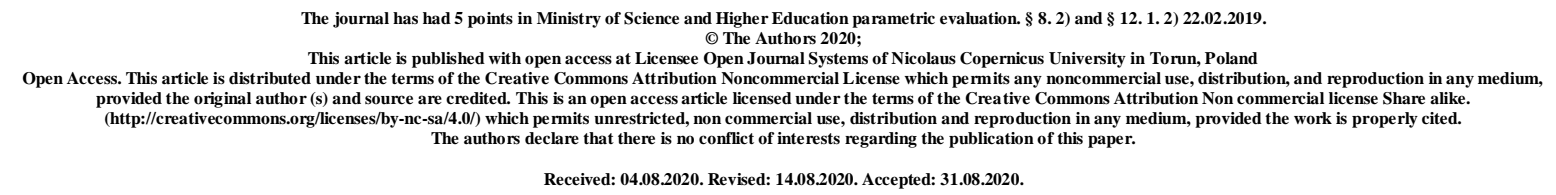

UDK 611.24:[617.55+616.748-005.1/-005.4]-092.9

\title{
MORPHOLOGICAL CHANGES IN LUNG TISSUE IN CASE OF BLUNT ABDOMINAL TRAUMA, MASSIVE BLOOD LOSS, AND ISCHEMIA- REPERFUSION OF THE LIMB
}

\author{
O. V. Fedoseeva \\ GO "Ukrainian Scientific and Practical Center of Emergency and Disaster Medicine \\ Ministry of Health of Ukraine", Kyiv, Ukraine
}

\section{Abstract}

Introduction. Combined trauma remains an urgent problem in medicine because it is the leading cause of death in victims of working age. Among the main causes of death in the prehospital stage among the injured is massive blood loss and further development of organ dysfunction, including lung. In recent years, significant progress has been made in providing assistance to victims of massive blood loss, but the impact of ischemia-reperfusion of the extremities in the development of systemic changes remains unresolved.

Objective of the research: to study morphological changes in lung tissue in the model of combined abdominal trauma, massive blood loss and ischemia - reperfusion of the lower extremities.

Materials and methods. To conduct the experimental study used 80 adult white male rats of the Wistar line weighing 190-220 g. The experimental animals were in the standard diet of vivarium.

The experimental animals were divided into the following groups: control and 3 
experimental ( 8 animals in each group). In animals of the first experimental group under thiopental-sodium disorder $\left(40 \mathrm{mg} \mathrm{kg}^{-1}\right)$ proximal to the lower limbs was imposed hemostatic plait for a period of $120 \mathrm{~min}$. In the second experimental group, a combined injury of the abdominal organs was simulated by applying two metered blows to the abdominal area; hypovolemic shock was modeled by blood flow from 20 to $22 \%$ of circulating blood volume from the femoral vessels. In the third experimental group, the combined injury of the abdominal organs, massive blood loss and reperfusion of the extremities was modeled. Animals in the control group were administered only in anesthesia.

The animals of the experimental groups were removed from the experiment under conditions of thiopental sodium anesthesia by total blood flow from the heart after 1, 3 and 7 days after modeling trauma. Pulmonary tissue was collected for the study.

For histological examination, the lower lobe of the right lung was removed, which was fixed in $10 \%$ formalin solution, after which the macrodrug was compacted in paraffin. Sections were obtained on a microtome followed by staining with hematoxylin eosin. After fixation of sections on individual slides studied the structure of the lungs in the study groups. Used equipment: LOMO Biolam I microscope; system of digital display of images of histological preparations. Particular attention in the study was paid to changes in the parenchyma and the main structural components of the lungs.

Results and Discussion. In the simulation of ischemia-reperfusion in the early periods in animals, changes were visualized from the first day in the form of endothelial lesions, increased perivascular edema, and cellular infiltration with signs of acute inflammation up to 3 days of experiment and formation of dyslectases up to 7 days of the experiment. When modeling closed abdominal trauma and massive blood loss in the lungs, structural changes increase from the first day of the experiment in the form of a sharp decrease in blood supply, lymphoplasmic infiltration of the stroma of the interalveolar septa, and the appearance of segmental leukocytes from 1 day of the experiment. After 3 days in the lumen of the vessels appear manifestations of erythrocyte aggregation and the formation of small parietal thrombi and pronounced dystrophic-necrotic changes in pneumocytes. After 7 days of the experiment, the acute manifestations are somewhat reduced, but there are pronounced dystrophic changes of the epithelium and pronounced lympho-plasmacytic infiltration of the stroma of the interalveolar septa, dystelectasis.

Closed abdominal trauma, massive blood loss, and ischemic-reperfusion syndrome in the early periods in the lungs were manifested by pronounced structural changes: thickening of the interalveolar septa due to intensive infiltration of lymphoplasmocytes, especially on 
day 3 of the experiment with a moderate inflammatory reaction. After 7 days of the experiment, the cellular and inflammatory infiltration of the stroma and dystrophic manifestations decreased slightly. The obtained data indicate the development of systemic changes in experimental animals and a significant increase in the possibility of developing multiple organ failure syndromes. This indicates the need for further study of the effects of reperfusion in the context of combined trauma and, accordingly, the development of effective means of correction.

Conclusions. Ischemia-reperfusion of the extremities alone and in combination with other injuries causes changes in lung tissue from the first day, progression to the third day of observation and a slight decrease in manifestations up to 7 days of the experiment. The results of the study suggest that ischemia-reperfusion is an important traumatic component in combined trauma.

Key words: reperfusion; experiment; lungs; morphological changes; abdominal trauma; massive blood loss.

Introduction. Combined trauma remains an urgent problem of medicine. It is the cause of death in victims of working age in all periods of the post-traumatic period [8]. Among the main causes of death at the prehospital stage among the injured is massive blood loss, at the hospital stage the development of systemic changes, manifested by organ dysfunction [9]. In recent years, significant progress has been made in providing assistance to victims of combined trauma and massive blood loss in both prehospital and hospital stages [8, $10]$.

An effective method of stopping bleeding in victims with massive bleeding is the use of tourniquets. It is known that the application of tourniquets for a period of two hours is safe, so it can cause the development of reperfusion ischemia and activation of the lipid peroxidation system [1-3,7]. Studies have shown that in the case of restoration of blood supply there is a multifactorial lesion of both ischemic and distant tissues.

On the other hand, victims with co-trauma have a significant risk of developing multiorgan failure syndrome, which develops against the background of shock and the occurrence of systemic inflammatory response syndrome. It is known that the pattern of its development, the sequence of functional organ disorders and the severity of the manifestation is determined by the nature of the damage and the reactivity of the organism. It is proved that the lungs are an important "target organ" in the development of this process, in fact they are the pacemaker of multiorgan failure $[4,5]$. Pathological changes in lung function remain an 
important factor in high mortality in victims of combined trauma. Thus, in one study in the development of acute respiratory distress syndrome, mortality ranged from 34.9 to $46.1 \%$ [6]. Of particular importance is the development of changes in the lungs of victims with combined trauma and massive blood loss, as massive transfusion of infusion solutions may be a factor in the development of changes in lung tissue.

However, risk factors and forecasts of individual components of multiorgan failure remain insufficiently studied.

In view of the above, the effect of reperfusion of the lower extremities on the development of systemic changes in combined trauma was studied in our experimental study.

The aim of this study was to study morphological changes in lung tissue in the model of combined abdominal trauma, massive blood loss and ischemia - reperfusion of the lower extremities.

Materials and methods. In the simulation of ischemia-reperfusion in the early periods in animals, changes were visualized from the first day in the form of endothelial lesions, increased perivascular edema, and cellular infiltration with signs of acute inflammation up to 3 days of experiment and formation of dyslectases up to 7 days of the experiment. When modeling closed abdominal trauma and massive blood loss in the lungs, structural changes increase from the first day of the experiment in the form of a sharp decrease in blood supply, lymphoplasmic infiltration of the stroma of the interalveolar septa, and the appearance of segmental leukocytes from 1 day of the experiment. After 3 days in the lumen of the vessels appear manifestations of erythrocyte aggregation and the formation of small parietal thrombi and pronounced dystrophic-necrotic changes in pneumocytes. After 7 days of the experiment, the acute manifestations are somewhat reduced, but there are pronounced dystrophic changes of the epithelium and pronounced lympho-plasmacytic infiltration of the stroma of the interalveolar septa, dystelectasis.

Closed abdominal trauma, massive blood loss, and ischemic-reperfusion syndrome in the early periods in the lungs were manifested by pronounced structural changes: thickening of the interalveolar septa due to intensive infiltration of lymphoplasmocytes, especially on day 3 of the experiment with a moderate inflammatory reaction. After 7 days of the experiment, the cellular and inflammatory infiltration of the stroma and dystrophic manifestations decreased slightly. The obtained data indicate the development of systemic changes in experimental animals and a significant increase in the possibility of developing multiple organ failure syndromes. This indicates the need for further study of the effects of reperfusion in the context of combined trauma and, accordingly, the development of effective 
means of correction.

Results and discussion. Histological examination of the lung after one day of reperfusion ischemia revealed structural changes in the vessels. Damage to the organization of endothelial cells and focal vasospasm were visualized in the lumens of arterial vessels. Perivascular edema was not observed. After three days of the experiment, the blood supply to the organ remained uneven, in some fields of vision increased. In the interalveolar septa there is an increase in lymphoplasmic infiltration and focal stromal edema. Among cellular elements, the number of neutrophilic granulocytes and mast cells, extravascular erythrocytes increased. Necrotized alveolocytes were observed in some areas of the alveoli. Examination of the lung after seven days of the experiment revealed a slightly reduced blood supply to the vessels of the microcirculatory tract, a sufficiently pronounced lympho-histiocytic infiltration of the interalveolar septa, a decrease or absence of stromal edema. Signs of dystelectasis increased, with the formation of small focal emphysema (Fig. 1). The acinuses remained free of exudate. The vast majority of epitheliocytes remained preserved, but there were cells with signs of protein dystrophy. The lumens of the small bronchioles did not contain exudate, but single leukocytes and a small amount of squamous epithelium were visualized.

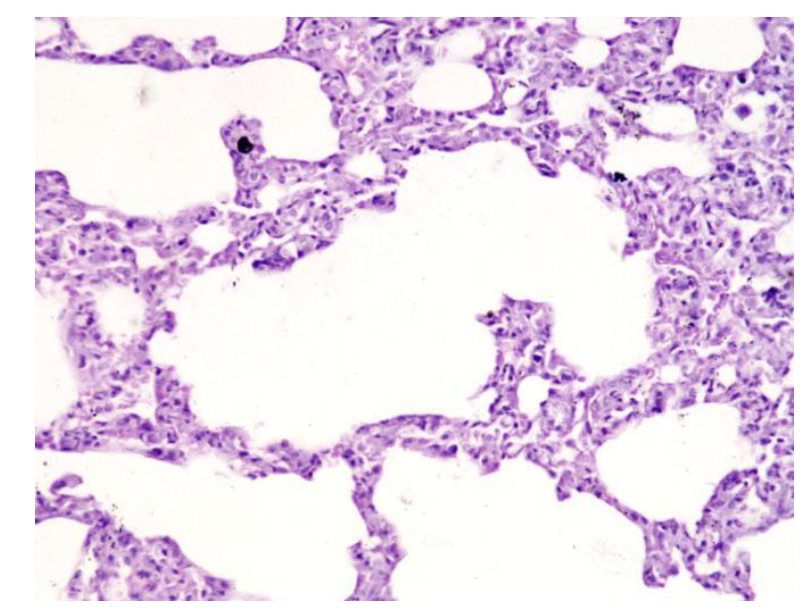

Fig.1. Pulmonary ischemia of limb reperfusion after 7 days. Areas of emphysematous expansion. Hematoxylin and eosin staining. Ex. $\times 200$.

When modeling abdominal trauma and massive blood loss in one day in the lungs visualized pronounced structural changes. The blood supply to small and medium-sized vessels was sharply reduced. Plasma with a sharply reduced content of erythrocytes could be observed in the lumens of blood vessels. Perivascular edema was not observed. After three days of the experiment, an increase in dystrophic-necrotic changes in the parenchyma was observed in the lungs. The blood supply to small and medium-sized vessels remained low. 
Dystrophically altered endotheliocytes were visualized in the vascular lumens, which is one of the factors of erythrocyte aggregation on the vessel wall and the formation of small parietal thrombi. Perivascular edema was not observed. After 7 days of the experiment, pronounced dystrophic and dystrophic-necrotic changes were observed in the lungs. The blood supply to small and medium-sized vessels remained low. Perivascular edema was not observed. The interalveolar membranes remained thickened due to significant infiltration of lymphocytes and plasma cells, but the number of segmental leukocytes decreased among cellular infiltrates. Intraplasmic inclusions, signs of severe protein balloon dystrophy, were observed in a significant part of pneumocytes (Fig. 2).

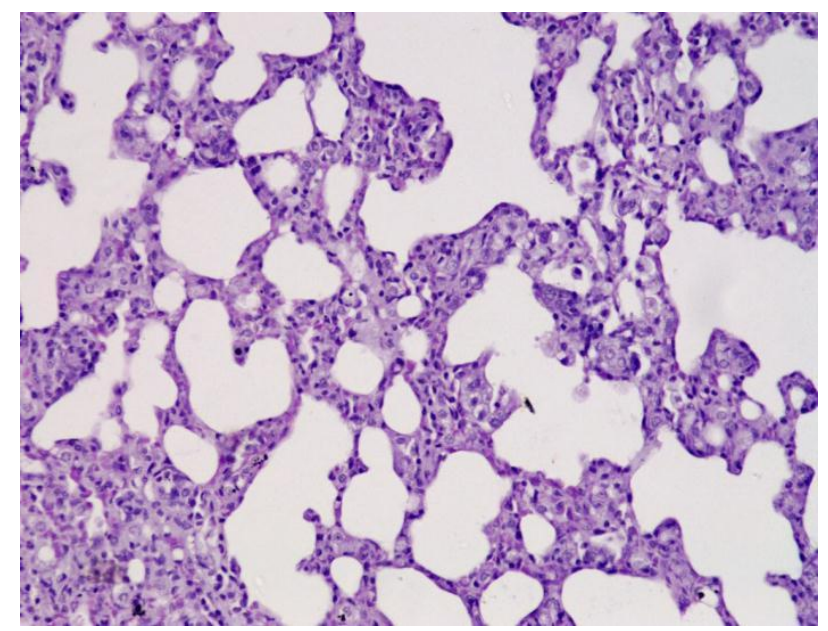

Fig. 2. Lungs in 7 days. Expressed balloon dystrophy of pneumocytes, significant thickening of the interalveolar septa. Hematoxylin and eosin staining. Ex. $\times 200$.

In the simulation of blunt trauma of the abdominal organs, massive blood loss and ischemia-reperfusion of the extremities after one day of the experiment in the lungs visualized pronounced manifestations. Blood supply to vessels of both small and medium caliber was uneven or reduced. The interalveolar membranes thickened mainly due to lymphoplasmic infiltration and the appearance of a small number of segmental leukocytes (Fig. 3). Stromal edema was not visualized. The alveolocytes are the vast majority preserved, some with signs of protein dystrophy. The alveoli dilated slightly, did not contain exudate.

After three days of the experiment, an increase in structural disorders was observed in the lungs. The blood supply of both small and medium vessels was increased mainly in the microcirculatory tract and was combined with diffuse erythrodiapadesis. Moderate edema with fibrin layers around larger vessels was visualized in the interalveolar stroma. There was a significant thickening of the interalveolar septa due mainly to lympho-plasmacytic 
infiltration and an increase in the number of segmental leukocytes (Fig. 4) with the formation of microabscesses. Stromal edema was insignificant, along with areas of fibrin accumulation around the arterioles. A significant part of alveolocytes remained preserved, but the number of dystrophically altered cells increased. The lumens of the alveoli were well visualized, some were significantly expanded, but did not contain exudate. Dystelectasis was observed.

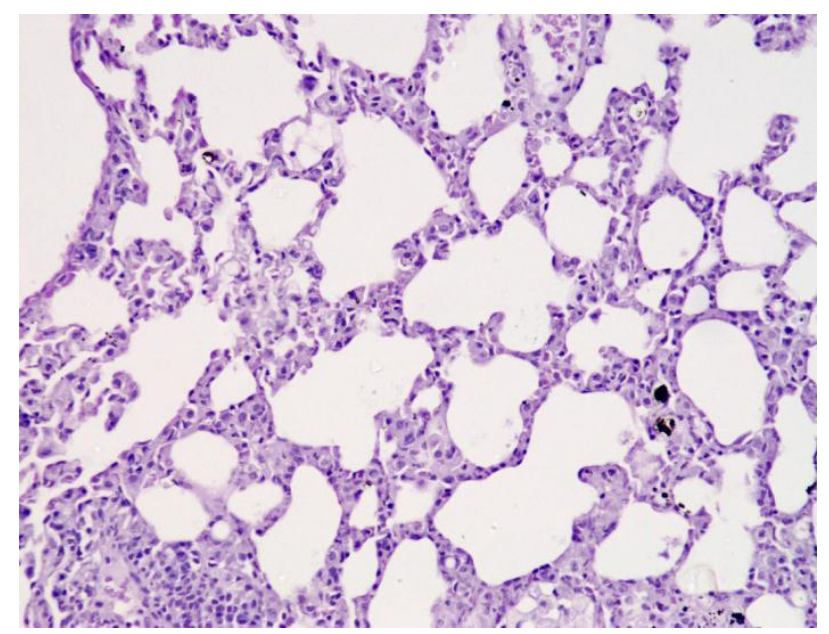

Fig. 3. Lungs in 1 day. Hematoxylin and eosin staining. Thickening of the interalveolar septa. Ex. $\times 200$

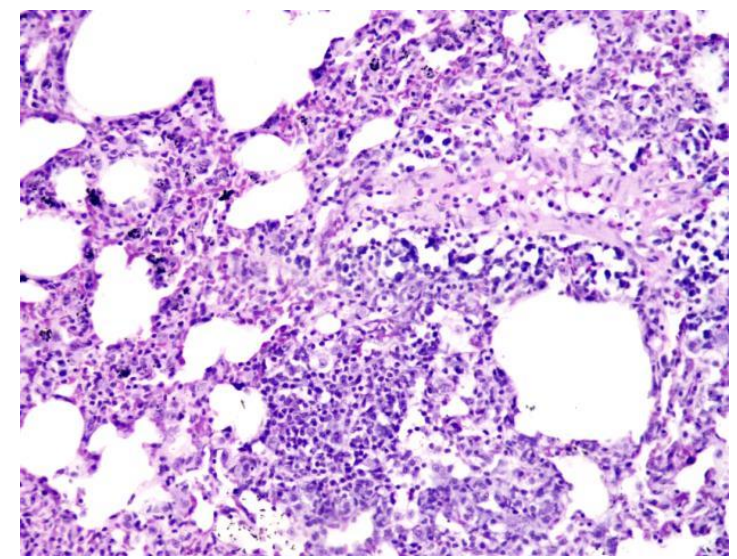

Fig. 4. Lungs in 3 days. Focal erythrodiapedesis, moderate stromal edema, leukocyte inflammatory infiltrates, dystelectasis. Hematoxylin and eosin staining. Ex. $\times 100$.

After seven days of the experiment, the blood supply to the lungs of vessels of both small and medium caliber was markedly reduced, compared with the previous group, their lumens were visualized, but did not contain erythrocytes. The interalveolar membranes remained somewhat thickened due mainly to lymphoplasmacytic infiltration, the number of segmental leukocytes decreased (Fig. 5). Stromal edema was observed in some fields of 
vision and was insignificant. The vast majority of alveolocytes remained preserved, but a significant part of them showed signs of protein, partly hydropic dystrophy. The alveoli in the vast majority remained moderately dilated, did not contain exudate.

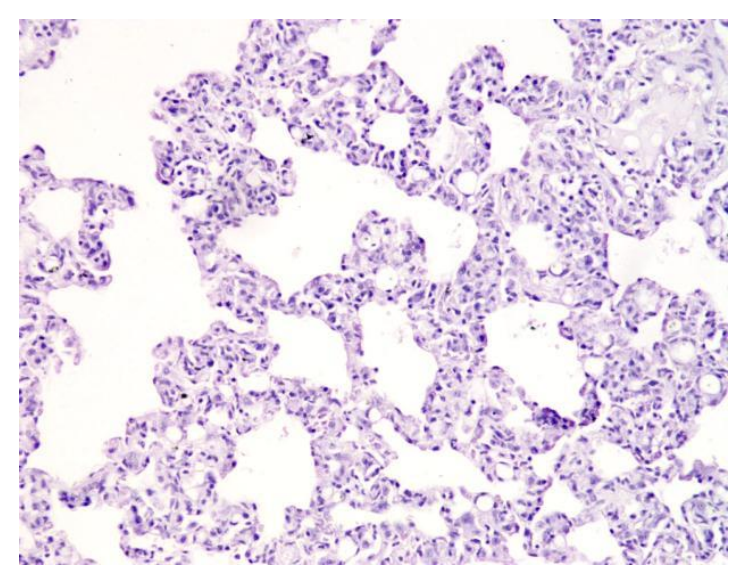

Fig. 5. Lungs after 7 days of the experiment. Hematoxylin and eosin staining. Ex. $\times$ 100.

Thus, in all groups of experimental animals were detected changes in lung tissue from the first day of the post-traumatic period. Our study proved that ischemia-reperfusion of the extremities significantly increases the pathological changes in lung tissue.

\section{Conclusions}

1. Ischemia-reperfusion of the extremities causes changes in lung tissue from the first day in the form of endothelial lesions, increased perivascular edema and cellular infiltration with signs of acute inflammation up to 3 days of the experiment and the formation of dyslectases up to 7 days of the experiment.

2. Injury of the abdominal cavity and massive blood loss causes changes in lung tissue with a maximum of manifestations in three days after the traumatic period and is manifested by aggregation of erythrocytes and the formation of small parietal thrombi and severe dystrophic-necrotic changes in pneumocytes.

3. Injury of the abdominal cavity, massive blood loss and ischemic-reperfusion syndrome in the early periods in the lungs were manifested by pronounced structural changes: thickening of the interalveolar septa due to intensive infiltration of lymphoplasmocytes, especially on the third day of the experiment with moderate inflammatory reaction. After seven days, the cellular and inflammatory infiltration of the stroma and dystrophic manifestations decreased slightly. 


\section{References}

1. A systematic review of the use of resuscitative endovascular balloon occlusion of the aorta in the management of hemorrhagic shock / J.J. Morrison, R.E. Galgon, J.O. Jansen at al. J Trauma Acute Care Surg. 2016. № 80. P.324-34.

2. Acute limb ischemia: contemporary approach/ I. Fukuda, M. Chiyoya, S. Taniguchi, W. Fukuda. The Journal of Thoracic and Cardiovascular Surgery. 2015. Vol.63, №10. P.540-548.

3. Administration of Curcumin Protects Kidney Tubules Against Renal IschemiaReperfusion Injury (RIRI) by Modulating Nitric Oxide (NO) Signaling Pathway / F. Liu, W. Ni, J. Zhang et al. Cell Physiol Biochem. 2017. № 44. P. 401-411.

4. Bone Marrow-Derived Mononuclear Cell Therapy Accelerates Renal IschemiaReperfusion Injury Recovery by Modulating Inflammatory, Antioxidant and Apoptotic Related Molecules / F. M. Ornellas, D. S. Ornellas, S. V. Martini et al. Cell Physiol Biochem. 2017. № 41. P. 1736-1752.

5. Effect of ischemia preconditioning on renal ischemia/reperfusion injury in rats / L. H. Fan, L. He, Z. Q. Cao at al. International Brazilian Journal of Urology. 2012. Vol. 38. P. $842-854$.

6. How I treat patients with massive hemorrhage / Johansson PI, Stensballe J, Oliveri $\mathrm{R}$ at al. Blood. 2014. T. 124(20). P.3052-3058.

7. Oxidative and nitrosative stress during pulmonary ischemia-reperfusion injury: from the lab to the OR/ J.F. Gielis, PAJ Beckers, J.J. Briedé et al. Ann Transl Med. 2017. Vol.5, №6. P.131.

8. Preventable death and interpersonal violence in the United States: Who can be saved? / H. Carmichael, L. Steward, E. D. Peltz et al. J Trauma Acute Care Surg. 2019. Vol. 87(1). P. 200-204.

9. Systematic review of prehospital tourniquet use in civilian limb trauma / D.Kauvar, M. Dubick, T. Walters et al. Journal of Trauma and Acute Care Surgery. 2018. Vol. 84. P. $819-825$.

10. Xiaoyu K. Chen, Christopher R. Rathbone, Thomas J. Walters Treatment of Tourniquet-Induced Ischemia Reperfusion Injury with Muscle Progenitor Cells. Journal of Surgical Research. 2011. Vol.170. 1. P. 65-73. 